\title{
Effect of Recycle Streams on Energy Performance and Closed Loop Dynamics of Distillation Sequences
}

\author{
S. Hernández ${ }^{\text {a, }}$, J.G. Segovia-Hernández $z^{\mathrm{a}}$, J. Carlos Cárdenas ${ }^{\mathrm{a}}$ and V. Rico-Ramírez \\ ${ }^{\text {a}}$ Facultad de Química, Universidad de Guanajuato, Noria Alta s/n, Guanajuato, Gto., \\ 36050, México \\ ${ }^{\mathrm{b}}$ Departamento de Ingeniería Química, Instituto Tecnológico de Celaya, \\ Av. Tecnológico y García Cubas s/n, Celaya, Gto., 38010, México
}

\begin{abstract}
This paper presents the retrofit of five conventional distillation trains for the separation of quaternary mixtures of hydrocarbons for feed compositions with high or low content of intermediate components. The retrofit implies the incorporation of liquid or vapour recycle streams among the conventional distillation columns. Each recycle stream removes one condenser or one reboiler. The introduction of thermal links can lower the energy consumption up to $40 \%$ in contrast to conventional distillation trains widely used in the industry. This efficiency in the use of energy is achieved because the recycle streams, introduced in the conventional distillation trains, reduce the remixing associated with higher energy consumption. Also, the introduction of recycle streams can improve the dynamic responses in contrast to those obtained in the conventional distillation sequences. Hence, the introduction of recycle streams in the conventional distillation sequences can improve both the energy consumption and the control properties.
\end{abstract}

Keywords: Distillation Sequences, Energy Savings, Dynamic Responses

\section{Introduction}

Conventional distillation trains are characterised by large demands of energy in the reboilers (Tedder and Rudd, 1978; Glinos and Malone, 1988; Fidkowski and Krolikowski, 1990); as a result, researchers and engineers are interested in developing new distillation arrangements that can reduce energy consumption. Most of those arrangements involves the use of thermally coupled distillation sequences (TCDS) which, in the case of the separation of ternary mixtures, can decrease the energy requirements around $30 \%$ in contrast to conventional distillation trains (Triantafyllou and Smith, 1992; Finn, 1993; Wolff and Skogestad, 1995; Hernández and Jiménez, 1996, 1999a, 1999b; Shah, 2002; Schultz et al., 2002). Thermal links reduce remixing in conventional distillation schemes, which contributes to the energy savings achieved in thermally coupled distillation sequences (Triantafyllou and Smith, 1992; Hernández et

*Author to whom correspondence should be addressed: hernasa@quijote.ugto.mx 
al., 2003). Nevertheless, mixtures with more than three components have not been studied to the same degree as ternary mixtures (Rong et al., 2003; Christiansen et al., 1997; Rong and Krawslaski, 2002). Therefore, in this paper we present a study of the retrofit of conventional distillation sequences for the separation of quaternary mixtures of hydrocarbons (A,B,C,D) with low or high content of intermediate components; such choice of the compositions was made because, in the case of ternary mixtures, the energy savings depend strongly on the amount of the intermediate component. The TCDS obtained are subjected to a study of their dynamic properties under the action of feedback control. It is well known that the separation of a quaternary mixture can be done using the five conventional distillation trains (King, 1980) shown in Figure 1. Figures 1A and 1B shown two classical distillation trains: the direct distillation train, in which the components are removed one by one in the overheads, and the indirect distillation train, in which the components are obtained one by one in the bottoms products. These sequences are widely used in industry. Conventional distillation trains can be changed into thermally coupled distillation sequences by removing a condenser and introducing a liquid recycle stream or eliminating a reboiler and adding a vapour recycle stream to the bottom of the column (Figure 2). For example, in the case of the conventional direct distillation train (Figure 1A), the reboiler of the first column is replaced by a recycle stream of vapour from the second column, and the reboiler of the second column is eliminated with a vapour recycle stream from the third distillation column. At this stage, recycle streams have been introduced, but it is necessary to optimise their flowrates in order to guarantee optimal energy consumption. Recycle streams are varied until the minimum energy requirement is detected, as reported in Hernández and Jiménez (1996, 1999b). The number of stages remains fixed in both types of distillation arrangements because in the retrofit design only recycle streams are introduced and condensers and reboilers are eliminated.

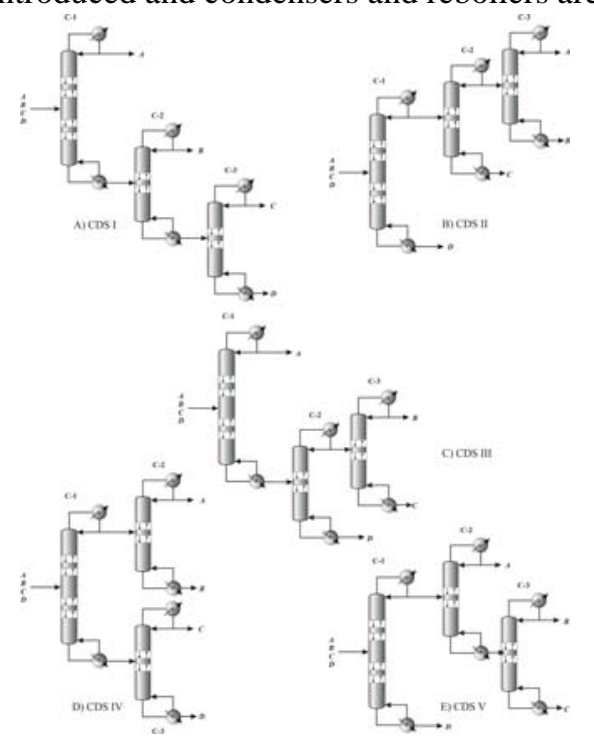

Figure 1. Conventional distillation sequences

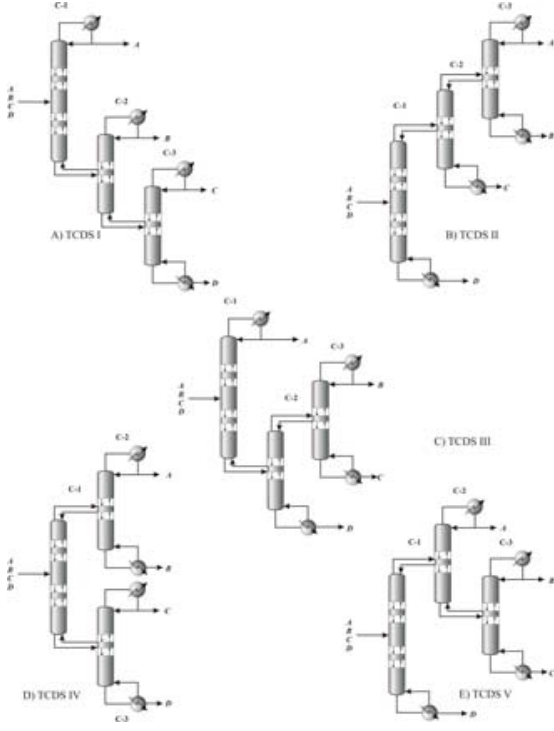

Figure 2. Thermally coupled distillation sequences 


\section{Case of Study}

We have explored energy consumption, remixing and closed-loop dynamic behaviour of the five conventional distillation sequences shown in Figure 1 and the corresponding thermally coupled distillation schemes of Figure 2, for the separation of two quaternary mixtures of hydrocarbons (Table 1) and two feed compositions (Table 2). The study was carried out via rigorous simulations by using the process simulator Aspen Plus $11.1^{\mathrm{TM}}$.

Table 1. Mixtures analysed.

\begin{tabular}{cc}
\hline Mixture & Components (A,B,C,D) \\
\hline M1 & n-pentane, n-hexane, n-heptane, n-octane \\
M2 & n-butane, i-pentane, n-pentane, n-hexane \\
\hline & \\
Table 2. Feed flowrates for each feed $($ lbmol/h). \\
\hline Feed & Flowrate (A/B/C/D) \\
\hline F1 & $30 / 20 / 20 / 30$ \\
F2 & $20 / 30 / 30 / 20$ \\
\hline
\end{tabular}

\section{Results}

\subsection{Energy}

In the first phase of the analysis, energy consumptions in the conventional distillation trains were obtained and compared (Table 3). For the case of mixture M1 and the two feed compositions, the CDS I (Figure 1A) was the most energy efficient; savings in energy of $37 \%$ and $11 \%$ for feed F1 and F2 respectively were obtained in comparison with the worst conventional distillation train. When the mixture M2 was considered, the sequences CDS V (Figure 1E) and CDS IV (Figure 1D) were the optimum options for the separation of feeds F1 and F2 respectively with savings of 58\% and $41 \%$.

In the second phase of the study, thermal links were introduced in the conventional distillation trains. As stated before, in order to obtain the optimum design in the use of energy, the interconnecting flows were varied until the minimum demand of energy was achieved. This task was completed with the process simulator Aspen Plus 11.1 ${ }^{\mathrm{TM}}$. In the optimisation procedure the interconnecting streams were changed one by one and the optimisation curves were obtained. The optimum energy consumptions found for the thermally coupled distillation trains are shown in Table 4. It can be observed, for the case of the separation of mixture M1 and the two feed compositions, that the TCDS I (direct thermally coupled distillation train) of Figure $2 \mathrm{~A}$ was the most efficient and presented energy savings of $27 \%$ in comparison to the CDS I scheme (direct distillation train). It is important to note that in these cases, the conventional direct scheme was the optimum sequence for both feed compositions when thermal links were introduced.

For the separation of mixture M2 and the two feed compositions, the optimum thermally coupled distillation trains presented savings of $40 \%$ and $30 \%$ in contrast to the conventional distillation sequences. 
In summary, the introduction of thermal links in the conventional distillation trains produced savings in energy of up to $40 \%$ in comparison with the optimum conventional distillation train.

Table 3. Energy consumptions (Btu/h) of the conventional distillation trains.

\begin{tabular}{|c|c|c|c|c|c|}
\hline Feed & CDS I & CDS II & CDS III & CDS IV & CDS V \\
\hline \multicolumn{6}{|c|}{ Mixture M1 } \\
\hline F1 & 3741914.2 & 3961719.8 & 5010941.41 & 3916616.7 & 5936384.3 \\
\hline $\mathrm{F} 2$ & 4064516.6 & 4558954.6 & 4251316.04 & 4226684.6 & 4596499.9 \\
\hline \multicolumn{6}{|c|}{ Mixture M2 } \\
\hline $\mathrm{F} 1$ & 8440246.2 & 6164263.7 & 10555639.9 & 4609531.7 & 4423507.7 \\
\hline $\mathrm{F} 2$ & 6741223.1 & 10555639.9 & 6359215.8 & 6174523.4 & 6944047.3 \\
\hline
\end{tabular}

Table 4. Energy consumptions (Btu/h) of the thermally coupled distillation trains.

\begin{tabular}{|c|c|c|c|c|c|}
\hline Feed & TCDS I & $\begin{array}{r}\text { TCDS II } \\
\text { M }\end{array}$ & $\begin{array}{l}\text { TCDS III } \\
\text { re M1 }\end{array}$ & TCDS IV & TCDS V \\
\hline F1 & 2712669.4 & 3070826.3 & 3688361.6 & 2788522.1 & 3141563.5 \\
\hline $\mathrm{F} 2$ & 2860660.9 & 3640303.3 & 3382957.9 & 2925223.3 & 3205683.6 \\
\hline \multicolumn{6}{|c|}{ Mixture M2 } \\
\hline F1 & 4318728.7 & 4596249.2 & 5544802.0 & 292522.3 & 2624437.5 \\
\hline $\mathrm{F} 2$ & 4400424.2 & 6935670.6 & 4946987.2 & 4448687.7 & 5714816.2 \\
\hline
\end{tabular}

\subsection{Remixing}

Remixing, presented naturally in conventional distillation trains, causes inefficiencies in the use of energy. These inefficiencies increase the energy of the reboilers required for the separation. In Figure 3 we observe the composition profiles for the four components in the first column of the CDS I for the separation of the mixture M1 and feed F1, as a typical example. The composition of component $\mathrm{B}$ increases until a maximum of 0.57 and then decreases to 0.28 in the bottom of the column, that is a consequence of remixing for component $\mathrm{B}$.

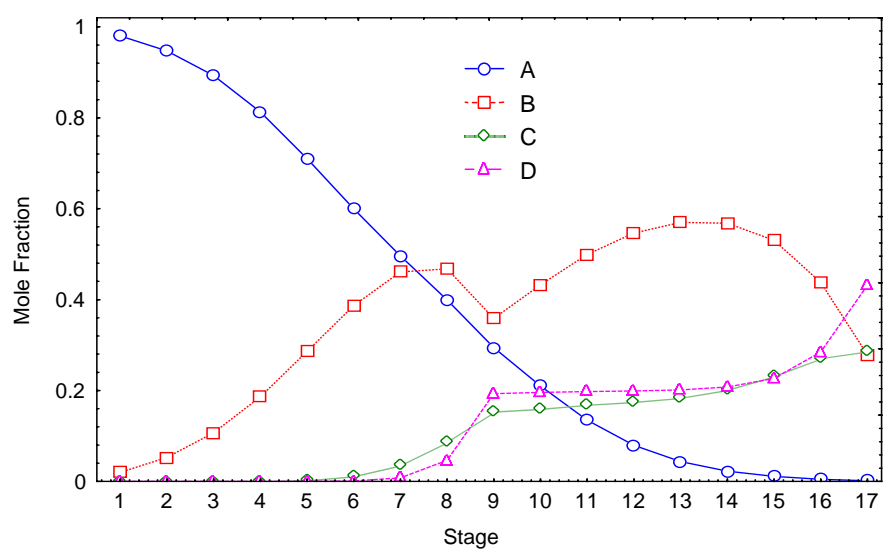

Figure 3. Remixing in the conventional distillation sequences 
A similar effect is presented in the composition of component $\mathrm{C}$ in the second column of the conventional distillation train CDS I. Having analysed all the mixtures and feed compositions, it was found that any conventional distillation column with at least one multicomponent product will have remixing.

\subsection{Dynamic behaviuor}

The optimum conventional and thermally coupled distillation sequences were subjected to a study of their dynamic responses under the action of feedback PI controllers. Changes in set points were considered and the optimal responses were chosen in terms of their IAE (Integral of the Absolute Error) values. Through the use of Aspen Dynamics $11.1^{\mathrm{TM}}$, the values of the controller gains $\left(\mathrm{K}_{\mathrm{c}}\right)$ and reset times $\left(\tau_{\mathrm{i}}\right)$ that provided a minimum value of IAE for a set point change for each separation scheme were computed.

Regarding the dynamic responses under the action of feedback controllers for changes in the set points, Table 4 showns the IAE values for a positive set point change in each product composition. TCDS I outperformed the dynamic responses of CDS I for set point changes in components $A$ and $B$ as shown in Table 4; however, for components $C$ and D, CDS I presented better values of the IAE. When a negative set point change of the same magnitude was implemented in each composition, a similar result was obtained. CDS I presented the best dynamic behaviour according to its lower values of the IAE (Table 4) in comparison to TCDS I for components B and D; on the other hand, when the set points of compositions of the products $\mathrm{A}$ and $\mathrm{C}$ were perturbed, TCDS I presented better dynamic responses than those obtained in CDS I.

Table 4. IAE results for set point changes in the distillation sequences.

\begin{tabular}{ccccc}
\hline \multirow{2}{*}{$\begin{array}{c}\text { Distillation } \\
\text { Sequence }\end{array}$} & $\mathrm{A}$ & \multicolumn{4}{c}{ Product stream } \\
\cline { 2 - 5 } & \multicolumn{4}{c}{ Positive change in set points } \\
\cline { 2 - 5 } & 0.0143109 & 0.0142759 & $\mathbf{0 . 0 0 0 1 0 4 2}$ & $\mathbf{0 . 0 0 0 4 5 9 3}$ \\
CDS I & $\mathbf{0 . 0 0 4 9 4 6 3 8}$ & $\mathbf{0 . 0 0 0 5 0 4 8}$ & 0.0049209 & 0.0099678 \\
TCDS I & \multicolumn{5}{c}{ Negative change in set points } \\
\hline & 0.00037819 & $\mathbf{0 . 0 0 0 2 3 6 4 5}$ & 0.0007281 & $\mathbf{0 . 0 0 0 0 7 4 5}$ \\
CDS I & $\mathbf{0 . 0 0 0 2 6 2 1 7}$ & 0.00107824 & $\mathbf{0 . 0 0 0 0 3 9 7}$ & 0.0003016 \\
\hline
\end{tabular}

\section{Conclusions}

The retrofit of conventional distillation trains was studied introducing thermal links for the separation of quaternary mixtures of hydrocarbons. Energy savings of up to $40 \%$ were obtained because the retrofit reduced the inefficiencies of the conventional distillation trains. This result is important because the retrofit involved only the introduction of recycle material streams. The recycle streams decreased significantly the remixing effects presented in the conventional distillation trains, associated with higher energy demands. Also, the dynamic responses of thermally coupled distillation 
sequences were as good as those obtained in conventional distillation sequences and, in some cases, the dynamic responses of thermally coupled distillation sequences were better than those of conventional distillation schemes. These results can establish that the energy savings are achieved without adding potential control problems. The lower control efforts (lower values of IAE) required by TCDS for some of the case studies indicate that these options may also provide a more efficient use of energy during its transient times.

\section{References}

Christiansen, A.C., Skogestad, S. and K. Lien, 1997, Comput. Chem. Eng., 21, S237.

Fidkowski, Z. and L. Krolikowski, 1990, AIChE J., 36, 1275.

Finn, A.J., 1993, Chem. Eng. Progress, 89, 41.

Glinos, K. and M.F. Malone, 1988, Chem. Eng. Res. Des., 66, 229.

Hernández, S. and A. Jiménez, 1996, Trans. Inst. Chem. Eng., 74, 357.

Hernández, S. and A. Jiménez, 1999a, Comput. Chem. Eng., 23, 1005.

Hernández, S. and A. Jiménez, 1999b, Ind. Eng. Chem. Res., 38, 3957.

Hernández, S., Pereira-Pech, S., Jiménez, A. and V. Rico-Ramírez, 2003, The Can. J. Chem. Eng., 81, 1087.

King, C. J., 1980, Separation Processes, $2^{\text {nd }}$ Edition, McGraw-Hill, New York, USA.

Rong, B.G., Kraslawski, A. and I. Turunen, 2003, Ind. Eng. Chem. Res., 42, 1204.

Rong, B.G. and A. Kraslawski, 2002, Ind. Eng. Chem. Res., 41, 5716.

Shah, P.B., 2002, Chem. Eng. Progress, 98, 46.

Schultz, M.A., Stewart, D.G., Harris, J.M., Rosenblum, S.P., Shakur, M.S. and D.E. O’Brien, 2002, Chem. Eng. Progress, 98, 64.

Tedder, D.W. and D.F. Rudd, 1978, AIChE J., 24, 303.

Triantafyllou, C. and R. Smith, 1992, Trans. Inst. Chem. Eng., 70, 118.

Wolff, E. A. and S. Skogestad, 1995, Ind. Eng. Chem. Res., 34, 2094.

\section{Acknowledgements}

The authors acknowledge financial support received from PROMEP and Universidad de Guanajuato, México. 